\title{
Adsorption behavior and current-voltage characteristics of CdSe nanocrystals on hydrogen-passivated silicon
}

\author{
Walzer, Karsten; Quaade, Ulrich; Ginger, D.S.; Greenham, N.C.; Stokbro, Kurt
}

Published in:

Journal of Applied Physics

Link to article, DOI:

$10.1063 / 1.1491016$

Publication date:

2002

Document Version

Publisher's PDF, also known as Version of record

Link back to DTU Orbit

Citation (APA):

Walzer, K., Quaade, U., Ginger, D. S., Greenham, N. C., \& Stokbro, K. (2002). Adsorption behavior and currentvoltage characteristics of CdSe nanocrystals on hydrogen-passivated silicon. Journal of Applied Physics, 92(3), 1434-1440. https://doi.org/10.1063/1.1491016

\section{General rights}

Copyright and moral rights for the publications made accessible in the public portal are retained by the authors and/or other copyright owners and it is a condition of accessing publications that users recognise and abide by the legal requirements associated with these rights.

- Users may download and print one copy of any publication from the public portal for the purpose of private study or research.

- You may not further distribute the material or use it for any profit-making activity or commercial gain

- You may freely distribute the URL identifying the publication in the public portal 


\title{
Adsorption behavior and current-voltage characteristics of CdSe nanocrystals on hydrogen-passivated silicon
}

\author{
K. Walzer ${ }^{\mathrm{a})}$ and U. J. Quaade \\ Mikroelektronik Centret (MIC), Technical University of Denmark, Building 345 East, \\ DK-2800 Lyngby, Denmark \\ D. S. Ginger and N. C. Greenham \\ Cavendish Laboratory, Madingley Road, Cambridge, CB3 OHE, United Kingdom \\ K. Stokbro \\ Mikroelektronik Centret (MIC), Technical University of Denmark, Building 345 East, \\ DK-2800 Lyngby, Denmark
}

(Received 6 March 2002; accepted for publication 8 May 2002)

\begin{abstract}
Using scanning tunneling microscopy and spectroscopy we have studied both the geometric distribution and the conduction properties of organic shell capped CdSe nanocrystals adsorbed on hydrogen-passivated $\mathrm{Si}(100)$. At submonolayer concentrations, the nanocrystal distribution on the surface was found to be highly nonhomogeneous, with an aggregation of most of the nanocrystals into islands of monolayer thickness. $I-V$ spectra collected on nanocrystals adsorbed on $n$ - and $p$-type substrates showed a strong difference in the conduction behavior, caused by the substrate: CdSe nanocrystals on $n$-Si:H caused a widening of the surface band gap by $1 \mathrm{eV}$ with respect to the gap of the substrate, while a significant narrowing of the gap was observed for nanocrystals on $p$-Si:H. This experimental result could be explained by modeling the system as a metal-insulatorsemiconductor (MIS) diode. Using this model we have found that the current through the MIS junction is limited by the nanocrystals only in one bias direction, while in the other bias direction the current is limited by the semiconducting substrate. This property may be of relevance for the construction of hybrid electronic devices combining semiconductor electrodes with nanoscale elements such as nanocrystals or organic molecules. (C) 2002 American Institute of Physics.
\end{abstract}

[DOI: $10.1063 / 1.1491016]$

\section{INTRODUCTION}

Over the past decade, semiconductor nanocrystals with diameters of a few nanometers have attracted much interest in the scientific community. This attention is based on the electronic properties of semiconductor nanocrystals, which combine semiconductor properties with quantum size effects. ${ }^{1,2}$ As a consequence, semiconductor nanocrystals are of interest both for fundamental studies and for use in a wide range of electronic and optoelectronic applications. Examples of electronic applications are tunnel diodes, ${ }^{3}$ lightemitting diodes, ${ }^{4}$ and photovoltaic cells. ${ }^{5}$ The sharp optical response of the nanocrystals also makes them suitable for "color-coded" biological labeling. ${ }^{6}$

Several attempts have been made to study the electronic properties of individual semiconductor nanocrystals, both by optical measurements using scanning confocal microscopy ${ }^{7}$ and by direct electrical approaches. Electrical measurements have included nanocontact experiments using an atomic force microscope (AFM) with an electrically conducting tip, ${ }^{8}$ nanogap experiments, ${ }^{9}$ scanning tunneling microscopy, ${ }^{10-13}$ and electrostatic force microscopy. ${ }^{14}$ Krauss and Brus showed by AFM measurements that CdSe nanocrystals capped with an organic shell of tri-n-octylphosphine oxide (TOPO) can be distributed homogeneously on a polymer surface. ${ }^{14}$ Using electrical force microscopy they found fur-

${ }^{a)}$ Electronic mail: kw@mic.dtu.dk ther that the nanocrystals could be positively charged by optical excitation.

For a wider use of semiconductor nanocrystals in electronic devices, however, knowledge about their behavior upon adsorption at semiconductor substrates is needed. Until now, the electrical properties of semiconductor nanocrystals were usually studied on substrates with ohmic conduction behavior, like gold or indium tin oxide (ITO). Self-assembly of nanocrystals from solution into semiconductor nanogaps may provide an attractive route for the fabrication of nanoelectronic devices with nanocrystals as the functional element. This article therefore aims to study the electrical properties of nanocrystals deposited from solution onto silicon substrates with different doping properties. We have found that the substrate doping plays a crucial role in the tunnel spectra of nanocrystals. For a better understanding of the measured $I-V$ curves we have calculated the electron transport through such a junction using the thermionic emission theory for metal-insulator-semiconductor (MIS) diodes and have compared it with the measured results.

\section{EXPERIMENT}

In this study we have used CdSe nanocrystals with a core diameter of $3.1 \mathrm{~nm}$, possessing an organic passivation shell of tri- $n$-octylphosphine oxide (TOPO). This shell prevents the nanocrystals from sticking together, and also isolates them electrically. The preparation of the nanocrystals 
was performed following the method of Murray et al. ${ }^{15}$ as modified by Katari and coworkers. ${ }^{16}$ The nanocrystals were stored in powder form under $\mathrm{N}_{2}$ atmosphere. Before use, excess TOPO was removed by repeated sonification in dry methanol followed by centrifugation. The nanocrystals were then dried under $\mathrm{N}_{2}$ flow and dissolved in toluene. Finally, the nanocrystal solution was filtered using a $0.45 \mu \mathrm{m}$ syringe filter to remove any particulate contamination. This final solution was stored in a $\mathrm{N}_{2}$ environment in the dark to prevent oxidation until it was used for adsorption on the silicon substrates.

For all experiments we used commercially available, highly doped $\operatorname{Si}(100)$ wafers with $n$ and $p$ doping. The wafers were antimony and boron doped, respectively, with a resistivity of $<0.025 \Omega \mathrm{cm}$ each (supplied by Okmetic, Finland). Scanning tunneling spectroscopy (STS) studies were performed on both $n$ - and $p$-type substrates, whereas the detailed studies of nanocrystal distribution were carried out only on the $n$-type substrates. Before use, all substrates were hydrogen-passivated by immersion in a 5\% HF solution for $45 \mathrm{~s}$, followed by a short rinse with deionized water. Such hydrogen-passivated silicon surfaces are hydrophobic and thus dry when taken out of the water. They can withstand oxidation at ambient conditions for some time, ${ }^{17}$ which makes it possible to prepare a clean $\mathrm{Si}(100)$ : $\mathrm{H}$ surface using wet-chemical techniques, and subsequently to transfer it into ultrahigh vacuum (UHV), without changing the passivation.

The nanocrystals were deposited onto the passivated $\mathrm{Si}$ substrates from a dilute toluene solution. The samples were made by applying a well-defined, microliter amount of this nanocrystal solution onto the substrate at room temperature. The amount of liquid was selected to be big enough to cover the whole substrate. The solvent evaporated within a few seconds. After the deposition of the nanocrystals, the samples were mounted and inserted into UHV within $15 \mathrm{~min}$.

Nanocrystal solutions with concentrations of $0.25 \mathrm{mg} / \mathrm{ml}$ and $0.005 \mathrm{mg} / \mathrm{ml}$ were used. Neglecting the effect of the TOPO shell, the expected coverage $\epsilon$ can be estimated from

$$
\epsilon=\frac{c V}{\rho A d} g
$$

where $c$ is the concentration of particles in toluene, $V$ the deposited volume, $\rho=5.81 \mathrm{~g} / \mathrm{ml}$ the density of CdSe, $A$ the sample surface area, $d=3.1 \mathrm{~nm}$ the diameter of the nanocrystals, and $g$ a geometric factor which equals 1 if we consider the nanocrystals to be small cubes and 1.5 if we consider their spherical shape. Using the more dilute solution with $c=0.005 \mathrm{mg} / \mathrm{ml}, \quad V=10 \mu \mathrm{l}, A=6 \times 6 \mathrm{~mm}^{2}$, and $g$ $=1.5$, the estimated particle density $\epsilon$ on the surface is about $12 \%$, corresponding to about 150 particles in an area of 100 $\times 100 \mathrm{~nm}^{2}$.

All scanning tunneling microscopy (STM) measurements were carried out with a room temperature UHV STM. ${ }^{18}$ We have used electrochemically etched tungsten tips which were cleaned in 5\% hydrofluoric acid immediately before insertion into UHV to remove tungsten oxide from the tip. Scanning electron micrographs were made using a LEO 1530 FEG scanning electron microscope. Some additional measurements were done using a Rasterscope 4000 atomic force microscope (AFM) ${ }^{18}$ This AFM was run in contact mode, using soft $\mathrm{Si}_{3} \mathrm{~N}_{4}$ cantilevers.

\section{IMAGING}

As a first step, we have collected STM pictures of the hydrogen-passivated silicon surface to detect the substrate roughness and its suitability as a substrate for nanocrystals of $3 \mathrm{~nm}$ in diameter. The result was that even after the HF etching, the silicon wafers show a very low roughness, which is less than $2 \mathrm{~nm}$ peak-to-peak in $>1 \mu \mathrm{m}$ cross section, with long ripples only. This roughness is quite acceptable for the application of $3 \mathrm{~nm}$ nanocrystals, since they should be easily distinguishable from the substrate.

The next prerequisite for a collection of spectroscopy data at individual nanocrystals is an overview of the sample surface and a selection of the particle for investigation. Therefore we have taken overview images of the samples by scanning electron microscopy (SEM), followed by STM pictures at a smaller scale. For the SEM investigation, the samples were produced as described, stored under $\mathrm{N}_{2}$, and imaged in the SEM within a few hours. Figure 1 shows such an overview of TOPO-capped CdSe nanocrystals on a $n$-type $\mathrm{Si}(100): \mathrm{H}$ substrate at different magnifications. Despite the small amount of material adsorbed on the surface, leading to low materials contrast, the nanocrystal aggregates can be clearly distinguished as bright areas against the dark background. It was not possible to resolve individual nanocrystals (although the instrument would have allowed it), possibly because of charging effects and sample degradation at high magnifications. It can be seen that the nanocrystals do not distribute homogeneously over the surface, but form irregularly shaped agglomerates of a few microns in diameter, see Figs. 1(b) and 1(c), similar to patterns observed in dendritic growth. The growth of agglomerates is consistent with a small energy of interaction between the nanocrystals and the $\mathrm{Si}: \mathrm{H}$ surface. In addition, the drying process of the droplet has left another, larger scale pattern: most of the nanocrystal clusters are arranged in rings around the perimeter of the drying droplet as shown in Fig. 1(a). This effect is well known, e.g., from the drying of a drop of spilled coffee on a table. ${ }^{19}$ Recently, such a behavior has also been reported for nanocrystals on glass substrates. ${ }^{20}$

Figure 2 shows STM images of a sample which was prepared using $2 \mu \mathrm{l}$ of the $0.005 \mathrm{mg} / \mathrm{ml}$ solution. Using this amount of material, we expect a nanocrystal density which is five times smaller than the one used in the SEM images. Indeed, on such samples it takes some time to find nanocrystals within the STM's scan range. Once they are found, three different areas can be recognized, as shown in Fig. 2(a). (A) shows the bare substrate, with a very low density of nanocrystals. (B) shows an area of well-packed nanocrystals, where individual nanocrystals cannot be resolved due to the blunt STM tip, which has a radius of curvature of about 20 $\mathrm{nm}$. (C) shows an intermediate area, where the nanocrystal distribution is rather homogeneous, so that features due to individual nanocrystals or small nanocrystal agglomerates can be resolved. Cross-section topography measurements re- 

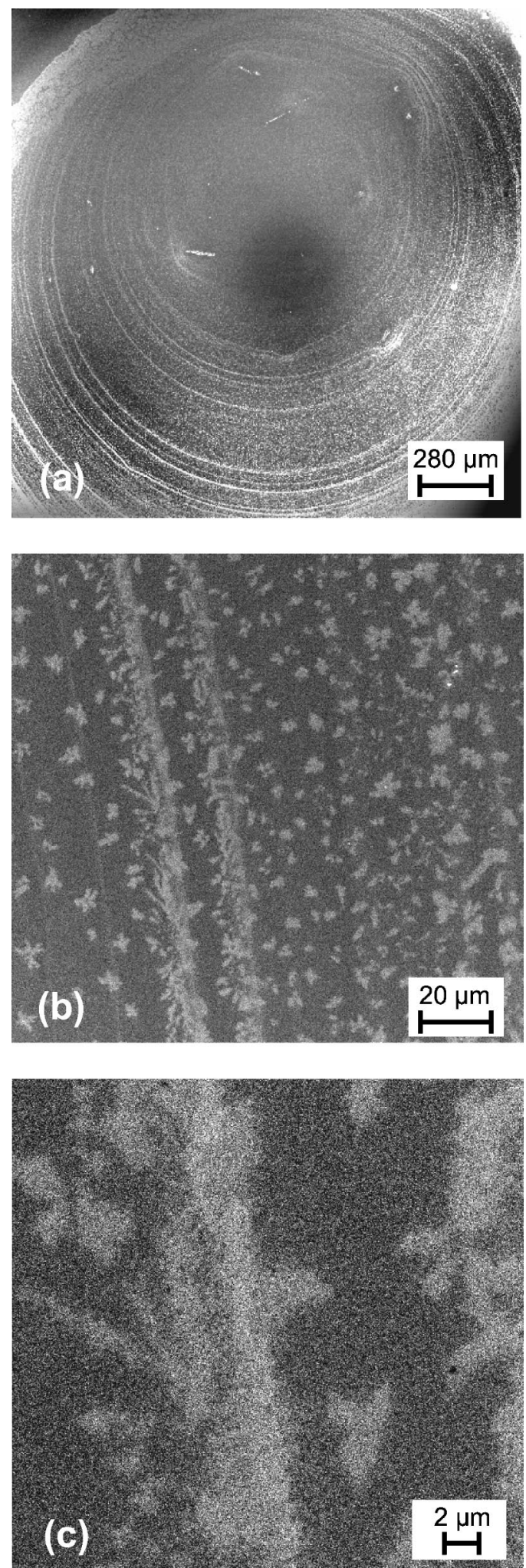

FIG. 1. Scanning electron micrograph of a $\mathrm{Si}(100)$ :H surface with an adsorbate of nominally 0.12 monolayer CdSe nanocrystals at different magnifications.

veal a structure height of typically $4 \mathrm{~nm}$, indicating a preferred monolayer coverage of the surface. We find that the pattern of surface coverage seen here is typical for a number of different samples on Si:H, including both (100) and (111) surfaces.
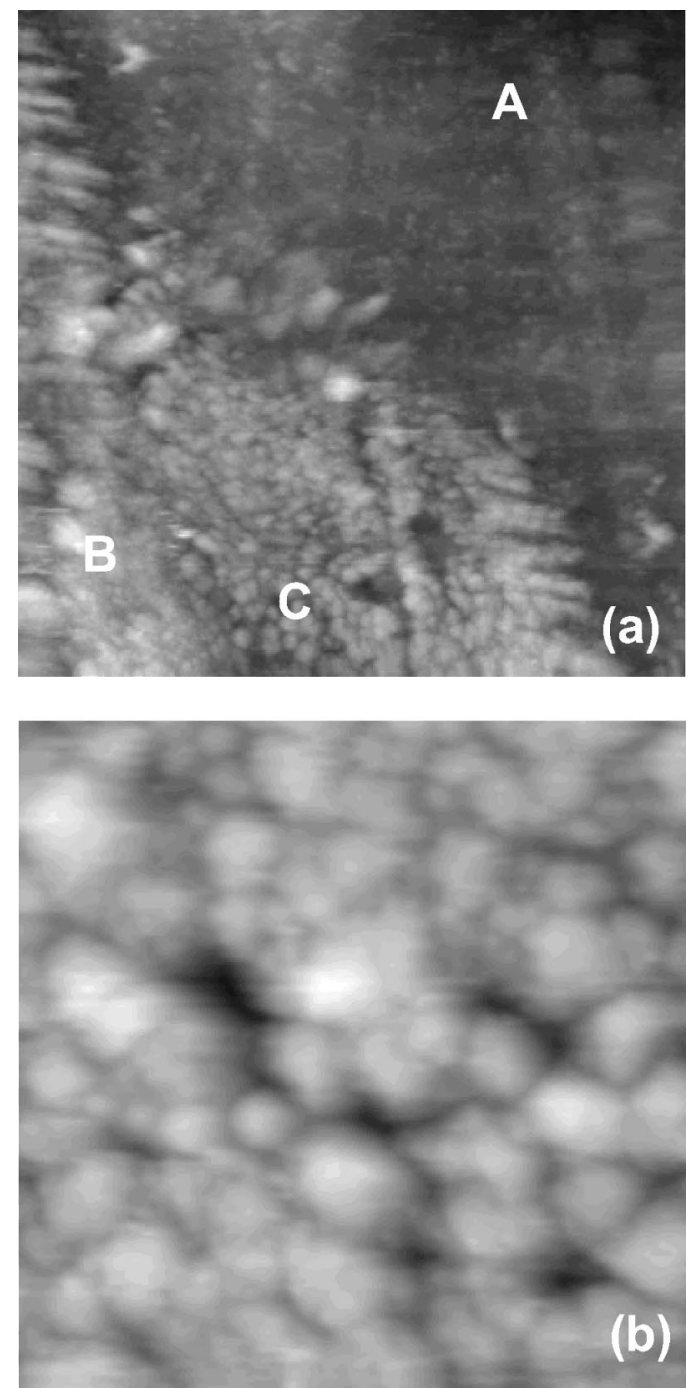

FIG. 2. (a) $\mathrm{Si}(100): \mathrm{H}$ surface with CdSe nanocrysals, $500 \times 500 \mathrm{~nm}^{2}$. The letters A-C mark the different typical areas. (b) shows a $100 \times 100 \mathrm{~nm}^{2}$ zoom into area C. Tunnel spectra were collected over small, clearly distinguishable particles. Both images: $U_{T}=-2.5 \mathrm{~V}, I_{T}=-100 \mathrm{pA}$, height scale $10 \mathrm{~nm}$.

AFM measurements with much bigger scan sizes (up to $20 \times 20 \mu \mathrm{m}^{2}$ ) confirmed the formation of such agglomerates. These measurements also show that the particles can be moved around the substrate by forces of $<1 \mathrm{nN}$, using contact mode AFM, consistent with weak binding of the particles to the surface. We do not believe, however, that the inhomogeneous particle distribution observed in the scanning probe measurements is due to the tip pushing isolated particles into agglomerates. Since isolated nanocrystals are not observed either in AFM with normal forces as small as $0.1 \mathrm{nN}$, nor in STM with tunnel currents $<50 \mathrm{pA}$, we are confident that the observed distribution is representative of the distribution immediately after deposition. Lowtemperature STM may in the future provide further information about nanocrystal motion on the substrate. For nanoscale devices fabricated and operated at room temperature, it is likely that nanocrystal agglomeration will be important in determining the final particle distribution on $\mathrm{Si}: \mathrm{H}$ surfaces. 

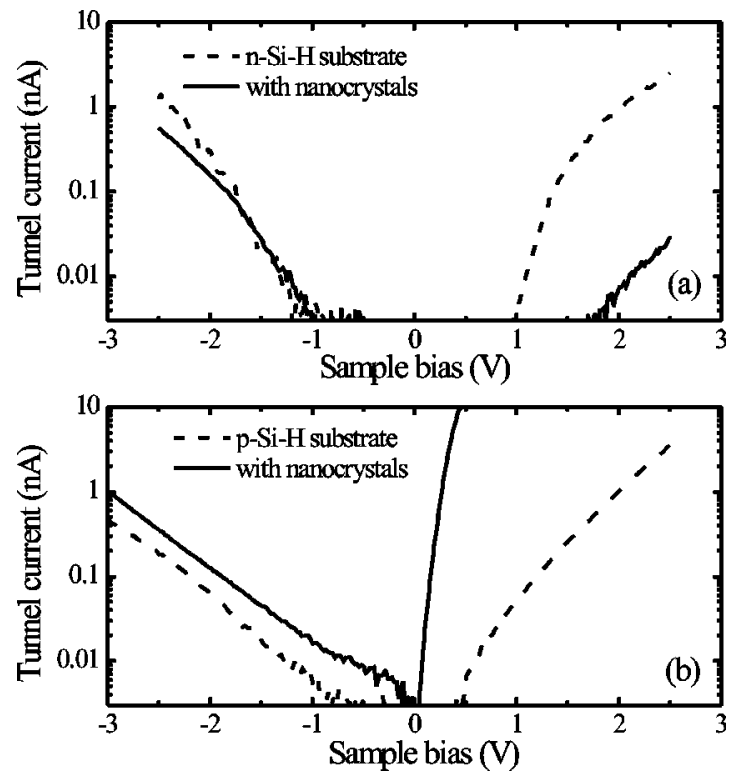

FIG. 3. $I-V$ spectra collected on (a) $n$-type and (b) $p$-type $\mathrm{Si}(100): \mathrm{H}$ samples, without and with CdSe nanocrystals applied. Identical samples were measured before and after nanocrystal self-assembly, using the same tip. In all cases, the feedback setpoint was $-2.5 \mathrm{~V}, 0.1 \mathrm{nA}$. The spectra were averaged over 20 successive curves without further filtering or selection.

Figure 2(b) shows a magnified image of the area (C) of Fig. 2(a). The nanocrystals appear broader than expected, which is caused by the blunt shape of the STM tip. These regions were used for the collection of tunnel spectra.

\section{TUNNELING SPECTROSCOPY}

Local $I-V$ spectra were collected on top of the nanocrystals in the intermediate density region [type (C) in Fig. 2(a)]. These areas are closest to the ideal case of isolated nanocrystals. The spectra were collected at room temperature during a short interrupt in the feedback loop. Thus the tipsample distance is held constant. For reasons of comparability, we have chosen the same current and voltage setpoint conditions for all spectra shown: $-2.5 \mathrm{~V}$ sample bias and -100 pA tunnel current. The curves shown in Fig. 3 have been acquired by averaging over 20 individual curves. This averaging reduced the noise in the spectra, while not changing the essential features.

Figure 3(a) shows the tunneling spectrum for a freshly passivated $n$-type $\mathrm{Si}(100)$ : $\mathrm{H}$ surface (dashed line) in a region where no nanocrystals are present. We observe a clear gap between -1.1 and $1.0 \mathrm{eV}$. In case of the $p$-type substrate, we observe a similar behavior [see Fig. 3(b), dashed line], although the gap is somewhat smaller. This is consistent with previous measurements on the $\mathrm{Si}(111): \mathrm{H}$ surface. ${ }^{21}$ Since hydrogen-passivated silicon possesses no surface states one might expect a gap identical to the bulk band gap of $\mathrm{Si}$, which is $1.16 \mathrm{eV}$. In fact, the measured band gaps are approximately twice this value due to band bending in the substrate caused by the electric field of the STM tip.

The solid lines in Figs. 3(a) and 3(b) show the $I-V$ spectra measured on the same substrates, but over nanocrystals. It is obvious that the adsorption of the nanocrystals has drastically changed the conduction behavior of the samples.
Also, the $n$ - and $p$-type substrates give very different results. With the $n$-type substrate the spectrum shows a clear gap, as on the bare substrate, however, it is much wider. The gap widens at positive sample bias to give a total gap width of $3.1 \mathrm{eV}$, ranging from -1.1 to $2.0 \mathrm{eV}$. With $p$-doped substrates, however, we observe completely different $I-V$ curves with a strong rectification. For the tip-sample distance given by the feedback values mentioned above, there is only a very small gap. If the current and voltage setpoint is changed, e.g., to $3.0 \mathrm{~V}, 100 \mathrm{pA}$, so that the tip moves significantly away from the sample, then even clearer diode characteristics are observed. The current rises uniformly with positive bias, and there is no detectable current at negative bias (up to $-5 \mathrm{~V}$ ).

\section{MODEL CALCULATIONS}

In this section we will analyze the spectroscopic data in terms of a simple theoretical model based on thermionic emission theory for metal-insulator-semiconductor (MIS) diodes. ${ }^{22}$ We will first introduce the model by calculating the $I-V$ characteristics of the bare $\mathrm{Si}: \mathrm{H}(100)$ surface, and afterwards extend the model to take into account the effect of nanocrystals on the surface.

\section{A. I- $V$ characteristics of the bare substrate}

In the following we will neglect the geometry of the STM tip and model the STM-semiconductor interface as an MIS diode. A similar model has previously successfully been used in Ref. 23 to describe STS data of a $p$-type $\mathrm{WS}_{2}$ surface. Materials parameters for silicon are taken from Sze, ${ }^{22}$ and we model the tip as a tungsten surface with an area of 1 $\mathrm{nm}^{2}$. We thus neglect the shape of the tip in modeling the electrostatic potential, and we might therefore slightly overestimate the bandbending effects. Since we do not know the exact shape of the tip there is some uncertainty in the tip work function, and we will use the value which gives the best agreement with the experimental data. We find that an optimal value is $W_{\text {tip }}=4.75 \mathrm{eV}$ which is slightly larger than the work function of the $\mathrm{W}(111)$ surface $(4.55 \mathrm{eV})$. The parameters of the model are summarized in Ref. 24.

Figures 4(a) and 4(b) show the results of the model for $n$-type and $p$-type substrates, respectively, with a tip-sample distance of $0.6 \mathrm{~nm}$. In the case of the $p$-type substrate we see that the model overestimates the current at negative bias, while the current is underestimated at positive bias. The slope of the $I-V$ curves agrees well with the measured data, however, the jump in the current at $1.3 \mathrm{~V}$ is not observed in the experimental data. Between -0.8 and $0.4 \mathrm{~V}$ the current becomes very small, since at these biases the Fermi level of the tip enters the band gap of silicon. In this bias regime, the current cannot be measured reliably by the STM, since the noise level of the instrument at the given conditions is 0.03 nA.

The dashed line in Fig. 4(b) shows the current carried by holes, which are the majority carriers in the substrate. At negative bias the substrate enters the inversion regime and the simple thermionic emission theory predicts that the main part of the current is due to the minority carriers (electrons). 

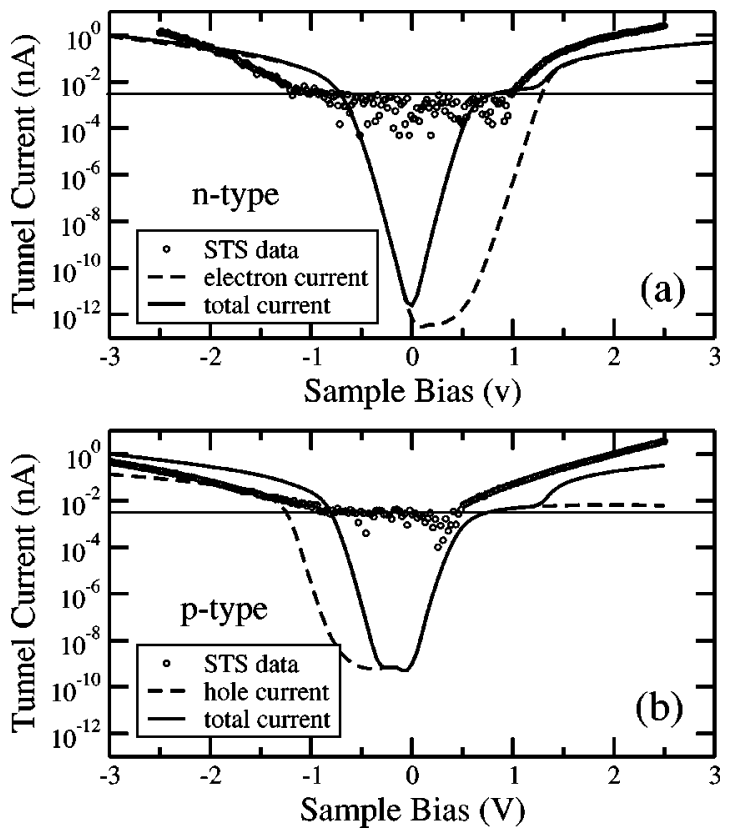

FIG. 4. Comparison of measured and calculated $I-V$ spectra for (a) $n$-type and (b) $p$-type hydrogen passivated silicon. The horizontal lines show the experimental noise level.

The low current observed in the experiment compared to the theory may be due to inefficient transport of minority carriers to the surface. It is likely that the surface is not in equilibrium with the bulk chemical potential in this regime, leading to a smaller current than predicted by the model.

For the $n$-type sample the slopes of the $I-V$ curves are in reasonable agreement with the experimental data, however, the absolute value of the current is overestimated at negative bias and underestimated at positive bias. In the voltage regime from -0.5 to $0.7 \mathrm{~V}$, the tip Fermi level is within the silicon band gap, and the current is therefore too small to be measurable. In this case it is the holes which are the minority carriers, and the surface enters the inversion regime at positive bias. Similar to the $p$-type sample we believe that within our model the minority current is overestimated in the inversion regime, however, for the $n$-type sample the hole current contributes very little to the total current at large positive biases.

\section{B. I- $V$ characteristics of the substrate with nanocrystals}

We now extend the one-dimensional MIS model to include the effects of the nanocrystals. In Fig. 5 we sketch our MIS model, with nanocrystals separated from the semiconductor substrate by the TOPO layer, and from the tip by the TOPO and a vacuum gap. In this simple model we use realistic values for the positions of the HOMO and LUMO levels in the nanocrystal, but we treat the density of states using an effective mass approximation. We thus neglect the effect of discrete energy levels due to electron confinement, and do not expect to be able to reproduce features due to resonant tunneling processes. We also neglect the charging energy of the CdSe particle, so our model will not show Coulomb blockade effects. Since the charging energy of our particles

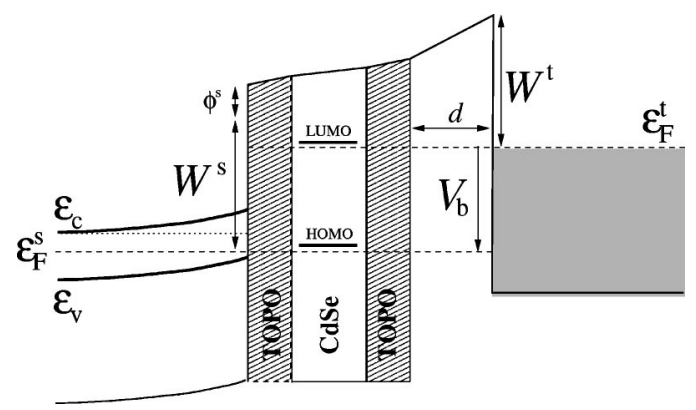

FIG. 5. MIS interface with CdSe nanocrystals on $p$-type silicon.

is expected to be approximately $0.2 \mathrm{eV}$ (significantly greater than $k T$ at room temperature), this is a major limitation in our model. Nevertheless, we expect to be able to reproduce the basic features of the interaction between the nanocrystals and the semiconductor substrate.

To obtain the relation between the silicon band bending, $\phi$, and the sample bias, $V_{b}$, we note that they are related through the electric field in vacuum, $E$, by

$$
\begin{aligned}
W_{T}+V_{b}= & W_{s}+\phi(E)+2 E d_{\text {topo }} / \epsilon_{\text {topo }} \\
& +E d_{\mathrm{CdSe}} / \epsilon_{\mathrm{CdSe}}+E d .
\end{aligned}
$$

The material parameters for CdSe are given in Ref. 25. From Fig. 5 we see that the electric field gives rise to a shift of the nanocrystal HOMO and LUMO levels relative to the semiconductor Fermi level $\epsilon_{F}^{s}$. The shift is given by

$$
\begin{aligned}
\epsilon_{\mathrm{HOMO}}(E)-\epsilon_{F}^{s}= & \epsilon_{\mathrm{HOMO}}^{0}-\epsilon_{F}^{s}+\phi(E)+E d_{\mathrm{topo}} / \epsilon_{\mathrm{topo}} \\
& +\frac{1}{2} E d_{\mathrm{CdSe}} / \epsilon_{\mathrm{CdSe}},
\end{aligned}
$$

where $\epsilon_{\mathrm{HOMO}}^{0}-\epsilon_{F}^{s}$ is the position of the HOMO relative to the semiconductor Fermi level for $E=0$. The factor of $\frac{1}{2}$ on the last term arises since we assume that the nanocrystal energy levels follow the average electrostatic potential in the CdSe layer. In Figs. 6(a) and 6(b) we show the biasdependent positions of the levels for the $n$-type and $p$-type substrates, respectively. We see that at zero bias the HOMO is slightly below the silicon valence band, while the LUMO is more than $1 \mathrm{eV}$ above the conduction band. The region between the dashed lines is the bias window, and the main contribution to the electron current comes from electronic states in this region. From this we see that the HOMO level is most important for the electron transport.

We next calculate the thermionic current, assuming that the electrons have to propagate via electronic states in the nanocrystal, rather than tunneling direct from substrate to tip. A simple WKB estimate of the direct tunneling current shows that this assumption is justified.

In Figs. 7(a) and 7(b) we show the results of our model for the $n$ - and $p$-type substrates, respectively. Since the absolute tip-sample distance is not experimentally measurable and might be different for the $n$ - and $p$-type substrates, we found that our model gives the best agreement with the experimental data if we use a tip-nanocrystal distance of $d$ $=0.5 \mathrm{~nm}$ for the $n$-type substrate and $d=0 \mathrm{~nm}$ for the $p$-type substrate (corresponding to the tip in contact with the TOPO layer). For the $p$-type substrate the model agrees well with 

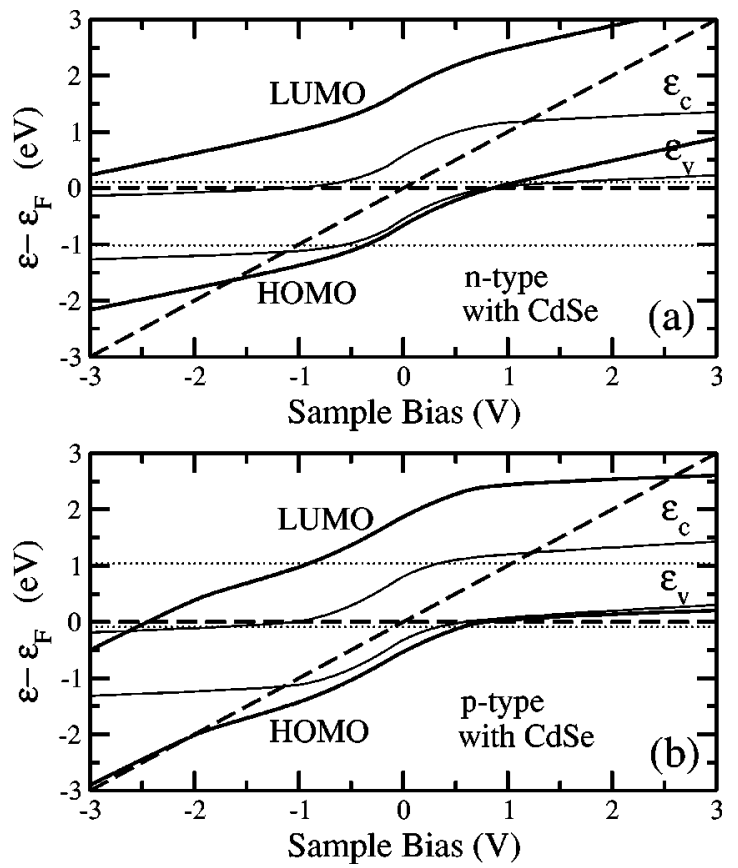

FIG. 6. Thin lines: position of the surface valence and conduction band edges relative to the bulk band edges as a function of sample bias for a MIS interface between tungsten and silicon, with CdSe nanocrystal in the insulator region. (a) $n$-type and (b) $p$-type silicon. Thick lines: position of the CdSe HOMO and LUMO levels. Dashed lines: sample and tip Fermi levels. Dotted horizontal lines: valence and conduction band edges in the bulk of the sample.

the STS data at positive sample bias. The large currents seen here in the accumulation regime at positive bias are consistent with the good alignment between the nanocrystal HOMO level and the silicon valence band. At negative biases, however, the current is greatly overestimated in the
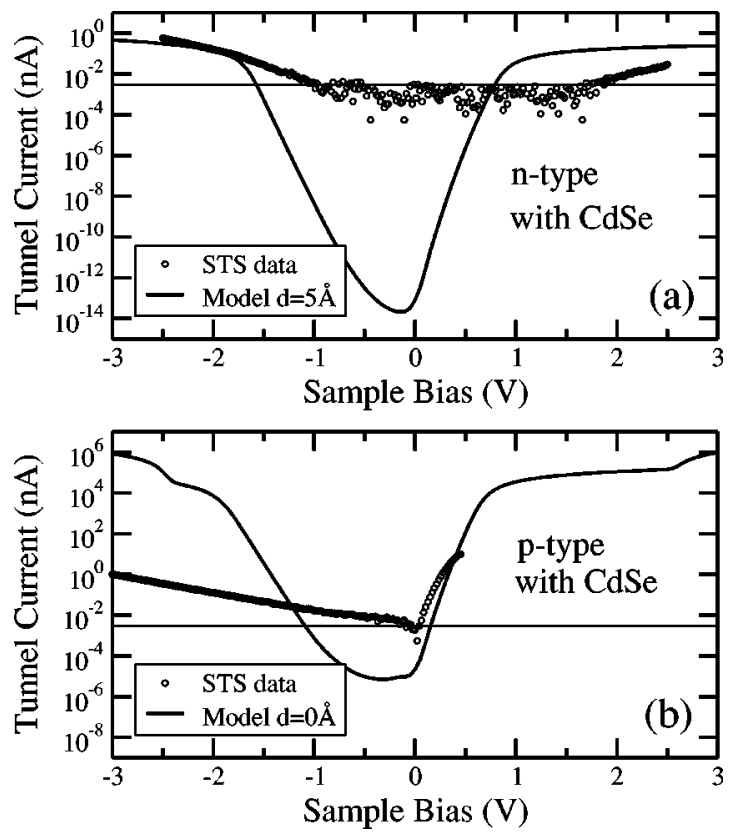

FIG. 7. Comparison of measured and calculated $I-V$ spectra for (a) $n$-type and (b) $p$-type H-passivated silicon with adsorbed CdSe nanocrystals of $d$ $=3 \mathrm{~nm}$ diameter. The experimental data were collected at $-3.0 \mathrm{~V}, 0.1 \mathrm{nA}$ (circles). The horizontal lines represent the noise level of the STM. model. For the $n$-type substrate the situation is reversed, with the current overestimated at positive bias. In both cases the discrepancies are found in the inversion regime, where the model predicts a high current of minority carriers which is not found in the STS data. We believe that this discrepancy may be due to the crudeness of the thermionic emission theory, which does not account for nonequilibrium effects in the inversion regime. These effects are associated with poor transport of minority carriers to the surface, leading to a lower density of surface carriers than predicted in our model.

Our results show that the use of a doped semiconductor substrate as opposed to a metal causes major differences in the measured electrical transport through the nanocrystals. On a metal substrate, the electronic properties of the nanocrystal dominate, and it is possible directly to measure the band gap of the particle and to resolve features due to Coulomb blockade and resonant tunneling effects. In our measurements, however, we cannot clearly identify features due to these effects at room temperature. Our modeling has shown that to understand the properties of nanocrystals on a semiconductor substrate, the effects of band-bending within the substrate must be taken into account. The measured voltage therefore contains a component which is due to the field within the substrate. This makes it harder to resolve Coulomb blockade and resonant tunneling effects in our measurements. The presence of other nanocrystals surrounding the particle under investigation may also obscure these effects. We note that interesting features, including negative differential resistance, are seen in the current-voltage characteristics of resonant tunneling diodes where a system with discrete quantum-confined energy levels is separated from heavily doped semiconducting leads by tunnel barriers. ${ }^{28} \mathrm{In}$ the future, it might be possible to reproduce these effects in our STM measurements by using substrates with higher doping levels.

\section{CONCLUSIONS}

We have studied the properties of monodisperse, TOPOcapped CdSe nanocrystals deposited at submonolayer coverage on hydrogen-passivated silicon substrates. SEM and STM measurements show that the particles tend to agglomerate into relatively close-packed domains of monolayer thickness. Reproducible tunnel spectra could be measured on top of identifiable individual nanocrystals within these domains. Tunnel spectra of nanocrystals with a diameter of 3 $\mathrm{nm}$ showed a $3.1 \mathrm{eV}$ wide conductance gap when deposited to hydrogen-passivated $n$-type silicon, whereas there was no conductance gap on $p$-type substrates. The basic features of our measurements can be reproduced by a simple thermionic emission model which includes the effects of band-bending in the substrate. It is important to take these semiconductor substrate effects into account in the design of future nanoelectronic devices where nanocrystals or conjugated molecules might be combined with semiconducting electrodes.

\section{ACKNOWLEDGMENTS}

The authors thank R. Lin and P. Thomsen for assistance and the Center for Surface Analysis of the Danish Techno- 
logical Institute for the collection of SEM pictures. This work was funded by the Danish Research Councils (STVF) and the European Commission within the 5th framework IST program under Contract No. IST-1999-10232.

${ }^{1}$ A. P. Alivisatos, J. Phys. Chem. 100, 13226 (1996).

${ }^{2}$ S. A. Empedocles, R. Neuhauser, K. Shimizu, and M. G. Bawendi, Adv. Mater. 11, 1243 (1999).

${ }^{3}$ S.-H. Kim et al., Appl. Phys. Lett. 74, 317 (1999).

${ }^{4}$ V. L. Colvin, M. C. Schlamp, and A. P. Alivisatos, Nature (London) 370, 354 (1994).

${ }^{5}$ B. O'Regan and M. Grätzel, Nature (London) 353, 737 (1991).

${ }^{6}$ M. Bruchez, M. Moronne, P. Gin, S. Weiss, and A. P. Alivisatos, Science 281, 2013 (1998).

${ }^{7}$ T. D. Harris et al., Semicond. Sci. Technol. 11, 1569 (1996).

${ }^{8}$ B. Alperson, S. Cohen, I. Rubinstein, and G. Hodes, Phys. Rev. B 52, R17017 (1995).

${ }^{9}$ D. L. Klein, R. Roth, A. K. L. Lim, A. P. Alivisatos, and P. L. McEuen, Nature (London) 389, 699 (1997).

${ }^{10}$ U. Banin, Y. Cao, D. Katz, and O. Millo, Nature (London) 400, 542 (1999).

${ }^{11}$ O. Millo, D. Katz, Y. W. Cao, and U. Banin, Phys. Rev. B 61, 16773 (2000).

${ }^{12}$ E. P. A. M. Bakkers and D. Vanmaekelbergh, Phys. Rev. B 62, R7743 (2000).

${ }^{13}$ P. Jiang, Z.-F. Liu, and S.-M. Cai, Surf. Sci. 486, L507 (2001).

${ }^{14}$ T. D. Krauss and L. E. Brus, Phys. Rev. Lett. 83, 4840 (1999).
${ }^{15}$ C. B. Murray, M. Nirmal, D. J. Norris, and M. G. Bawendi, Z. Phys. D: At., Mol. Clusters 26, 231 (1993).

${ }^{16}$ J. E. B. Katari, V. L. Colvin, and A. P. Alivisatos, J. Phys. Chem. 98, 4109 (1994).

${ }^{17}$ V. G. Lifshits, A. A. Saranin, and A. V. Zotov, Surface Properties on Silicon: Preparation, Structures, and Properties (Wiley, Chichester, 1994), pp. 227-242.

${ }^{18}$ Made by Danish Micro Engineering (DME).

${ }^{19}$ R. Deegan et al., Nature (London) 389, 827 (1997).

${ }^{20}$ S. Maenosono, C. Dushkin, S. Saita, and Y. Yamaguchi, Langmuir 15, 957 (1999).

${ }^{21}$ M. McEllistrem, G. Haase, D. Chen, and R. Hamers, Phys. Rev. Lett. 70, 2471 (1993)

${ }^{22}$ S. M. Sze, Semiconductor Devices (Wiley, New York, 1985).

${ }^{23}$ C. Sommerhalter, T. W. Matthes, J. Boneberg, P. Leiderer, and M. C. Lux-Steiner, J. Vac. Sci. Technol. B 15, 1876 (1997).

${ }^{24}$ Parameters used to describe the silicon-tip tunnel junction: $N_{D}$ $=10^{18} \mathrm{~cm}^{-3}, \quad m_{h}=0.5, \quad m_{e}=1, \quad k T=0.025 \mathrm{eV}, \quad W_{t}=4.75 \mathrm{eV}, \quad A_{\text {tip }}$ $=1 \mathrm{~nm}^{2}, d=0.6 \mathrm{~nm}, \chi=4.05 \mathrm{eV}, \epsilon_{g}=1.13 \mathrm{eV}, \epsilon_{0}=\epsilon_{v}-10 \mathrm{eV}$, and $\epsilon_{\mathrm{Si}}$ $=11.8$.

${ }^{25}$ Material parameters used to describe the CdSe nanocrystals: $m_{h}=0.45$, $m_{e}=0.13, \quad k T=0.025 \mathrm{eV}, \quad d_{\mathrm{CdSe}}=3.1 \mathrm{~nm}, \quad d_{\mathrm{topo}}=1.0 \mathrm{~nm}, \quad \epsilon_{\mathrm{HOMO}}$ $=-5.33 \mathrm{eV}, \epsilon_{\mathrm{LUMO}}=-2.93 \mathrm{eV}, \epsilon_{\mathrm{CdSe}}=9.2, \epsilon_{\mathrm{topo}}=2.5$. The values for $\epsilon_{\mathrm{HOMO}}$ and $\epsilon_{\mathrm{LUMO}}$ are taken from Ref. 26 and the value for $\epsilon_{\mathrm{topo}}$ is taken from Ref. 27. All other parameters are from Ref. 22.

${ }^{26}$ C. Wang, M. Shim, and P. Guyot-Sionnest, Science 291, 2390 (2001).

${ }^{27}$ J. Jiang, T. D. Krauss, and L. Brus, J. Phys. Chem. B 104, 11936 (2000).

${ }^{28}$ K. Shinohara et al., J. Cryst. Growth 175-176, 924 (1997). 